\title{
Developing Content for Migrants at Crossroads: Lessons Learned from Piloting an Online Community Portal for Overseas Filipinos
}

\section{Francis Raymond Calbay, HayPinas.org, Philippines}

\begin{abstract}
Millions of Filipinos have migrated to other countries to resettle and find work, forming one of the largest diaspora in contemporary times. But even with the ubiquity of the Internet, overseas Filipinos still complain about the lack of quality information sources for their myriad concerns (Del Rosario, 2007). This case study reports on the lessons learned from the pilot year of producing and managing content of HayPinas.org, an online community portal for overseas Filipinos. Taking cue from the trend of media convergence, this study seeks to outline a process model for developing content across online media platforms in the service of migrant advocacy. The study shares practical insights from HayPinas.org's experience in content development, online publishing, and social media management that is attuned to the information needs of its target audience.
\end{abstract}

Keywords: Media Convergence; Content Development; Social Media; Online Publishing, Overseas Filipinos 


\section{Background}

Overseas Filipinos are among the largest diasporic communities in the world today. Roughly 10 million of the population - one in ten or some 25 percent of the adult workforce - has left the Philippines to find jobs and settle abroad (Commission on Overseas Filipinos, 2010). Central bank data for 2011 indicate that these migrants contributed some \$18 billion US dollars in remittances to the Philippine economy. Since the government-facilitated mass labor exodus in the 1970 's, the number of Filipinos departing to temporarily or permanently resettle abroad has steadily increased. The trend continues despite the grim reality that migrants typically encounter such as racism, abusive labor conditions, and unequal rights as they relocate to a foreign country (Ayalon, 2009). But from mostly working menial jobs such as domestic and construction work, an increasing segment of overseas Filipinos now also hold management and specialist positions (Go, 2003). Indeed, Filipinos can be found in countries from Australia to Zimbabwe - in search of the proverbial greener pasture even if they sometimes find themselves in a new land that is poorer or much less safer than their own.

With their facility of the English language (a legacy of the American occupation of the islands) and their mixed foreign cultural influences, it is a common perception that Filipinos could better adjust in other countries compared to other migrant groups. However, Ofreneo and Samonte (2005) cite "social and cultural adaptation problems" (p. 8) as a persistent complaint of Filipino migrants. Another consistent challenge is the lack of information sources that address the many concerns that they face - from learning about laws, migrant rights, and current events. (Del Rosario, 2007). This is particularly surprising since there is a high penetration rate of Internet use among Filipinos: 83.1 percent log on to social networks, while 90.3 percent use blogs, as cited by Figer (2009: p. 279). There is also a dearth of studies on overseas Filipinos and new media use. As Paragas (2006) observes, "This is an important 
oversight since it is in the personal uses of global networks that transnational communication can be observed" (p. 50).

Against this backdrop, this research looks at the case of HayPinas.org, an online community portal dedicated to producing content on the issues and challenges faced by overseas Filipinos. The study aims to identify lessons learned from HayPinas.org's first year of operation and seeks to outline a practical process model on developing responsive content for and about Filipino migrants. In this study, responsive content is taken to mean as content that addresses and accounts for the perceived information needs, preferences, and feedback of its target audience. The rest of the paper is organized as follows: through a review of related literature, the concept of media convergence is explored, along with how it allows for a more participative model of communication that can give voice to disadvantaged social groups, such as migrants. Then, a case study of HayPinas.org takes center stage by categorizing published content and reviewing web analytics during the community portal's pilot year. With added data from the personal account of the HayPinas.org founder, lessons learned are summarized in creating and managing the community portal. Finally, the study concludes with a discussion on HayPinas.org's successes against its stated mission, and as well as with recommendations to continue its advocacy.

\section{Converging Media and Democratizing Cyberspace}

Before widespread Internet adoption, overseas Filipinos typically turned to personal communications and traditional media for the latest updates and news on the country they left behind (Del Rosario, 2007). But in what is touted as the rise of the "social web", alternative online media channels such as blogs, online forums, and social networks have increased in usage and popularity. For one, Komito and Bates (2011) observe "a trend away from friends 
and family and to the Internet as a source of information" (p. 292) when migrant Filipinos already residing in a foreign country decide to relocate. Although at most times fragmented, content from social media discusses important citizen and consumer issues. Yao (2010) observes that migrant Filipino women post blogs to share their experiences in the host country and to also recount memories from the homeland. Figer and De Torre (2012) find out that anonymous forum postings allowed for an exchange of information among overstaying or illegally working Filipinos abroad. These kinds of content bring out a wider array of anecdotes, points of view, and other narratives than what can be found from conventional media products.

Without the drive to profit, bottom-up grassroots media initiatives from individuals belonging to smaller social and cultural groups are a welcome contrast to traditional media that are typically controlled by corporations (Preston \& Kerr, 2001). For the first time in history, Castells (2005) observes that people are communicating messages and producing content in a mass medium that doesn't require traditional gatekeeping. The roles of media producer and audience are blurring with the content explosion on the Internet. Bowman and Willis (2003) use the term "We Media" to describe how the Web has provided a platform to serve the interests of private individuals and groups through readily available and easy to set up content creation tools. Rather than cater to a mass audience, content in social media can also directly address specific segments, such as migrants. Content created through social media can be cross-posted and rehashed in other channels as well, making it more shareable and searchable online and amplifying its reach and possible impact to the right audience. Indeed, the private and personal voice can matter more than ever, especially if it resonates to niche audience segments.

The social web takes the concept of media convergence further, where its basic form was 
previously understood as how audiences respond to a print article or a broadcast program through other means of communication such as emails. With the rise of personal computers as an increasingly preferred tool for media access, viewing content, posting reactions, and leaving other traceable user behavior on a single device are further results of convergence (Hartley, 2002: 39). Moreover, social media content - be it text, image, video or hybrids in between - is poised to diffuse on devices other than computers such as location-aware mobile devices and Internet-connected TVs. Jenkins predicts that "media will be everywhere and we will use all kinds of media in relation to each other" (2004: 34). Klinenberg (2005), notes that digital communication infrastructures are crucial for this level of convergence, and the fast rate of technological development heralds an unprecedented opportunity for more private individuals to participate.

Content from social media is fast becoming one of the preferred sources of information rather than as a mere alternative to traditional media products. As Bowman and Willis (2003) observe, "Faced with an overwhelming flow of information from a massive number of media sources, people are increasingly going to online communities to learn how to make sense of things." (p. 40). However, such online-based communication model is not devoid of criticism and challenges. For one, Herring (2004) notes that for the generation born with computers and mobile technology as just an everyday necessity, the novelty and utopian promises of the Internet might be underappreciated or even lost. The capacity-building potential of online communication persists in empowering marginalized groups such as low-income families and minorities (Mehra and Merkel, 2004). But the sad fact remains that basic computer literacy is not possessed by all, and Internet access may even be disallowed to migrant workers (Orange, Seitz \& Kor, 2012). The digital divide must be bridged especially as social media emerge as an important platform for information that could directly improve the quality of life. 


\section{HayPinas.org: Migrant Advocacy through Online Content}

HayPinas.org (http://www.HayPinas.org) labels itself as a "community portal for a new breed of overseas Filipinos" with an established online presence as a website and as a social network community. Launched in August 2011, this not-for-profit web project aims to reach out and serve the information needs of migrant Filipinos, particularly the 21 to 40 -year-old bracket who are engaged in professional occupations. Content is mostly in the English language, taking into account that the Philippines - an archipelago of some 7,000 islands - has hundreds of local dialects. New content is produced at a biweekly schedule, sometimes with more frequency. Content management is done in Taiwan where the core team (composed of overseas Filipinos themselves) is based, while content contributions come from community members staying in a few other countries. Reviewing the first year pilot of HayPinas.org, the primary sources for the case study included a content inventory, internal process documents and web analytics, and interview data from the HayPinas.org founder.

The primary platform for publishing content remained to be the website. Content typically took the form of text-based articles, photos (mostly as a supplement to the articles, some as photo essays), and videos. Features of the website included a blog-based homepage, post commenting and sharing functions, a discussion board area, and a collection of relevant links (including news feeds from around the Web) for overseas Filipinos. Labels were organized as tabs, for example: "Life Abroad", "Money", and "Migration Issues". Another key content channel was the YouTube account (http://www.youtube.com/haypinasorg) - linked from the website as the "Videos" tab - with videos recorded as tutorials and commentaries. For sharing content, two primary social networks were used: Facebook (http://www.facebook.com/haypinas) and Twitter (http://www.twitter.com/haypinasorg). The Facebook page also separately published polls and other announcements, while the Twitter 
account posted links to news and features of interest to overseas Filipinos. Website visitors were encouraged to share the content in popular social networks through a plugin that offered different levels of graphic badges.

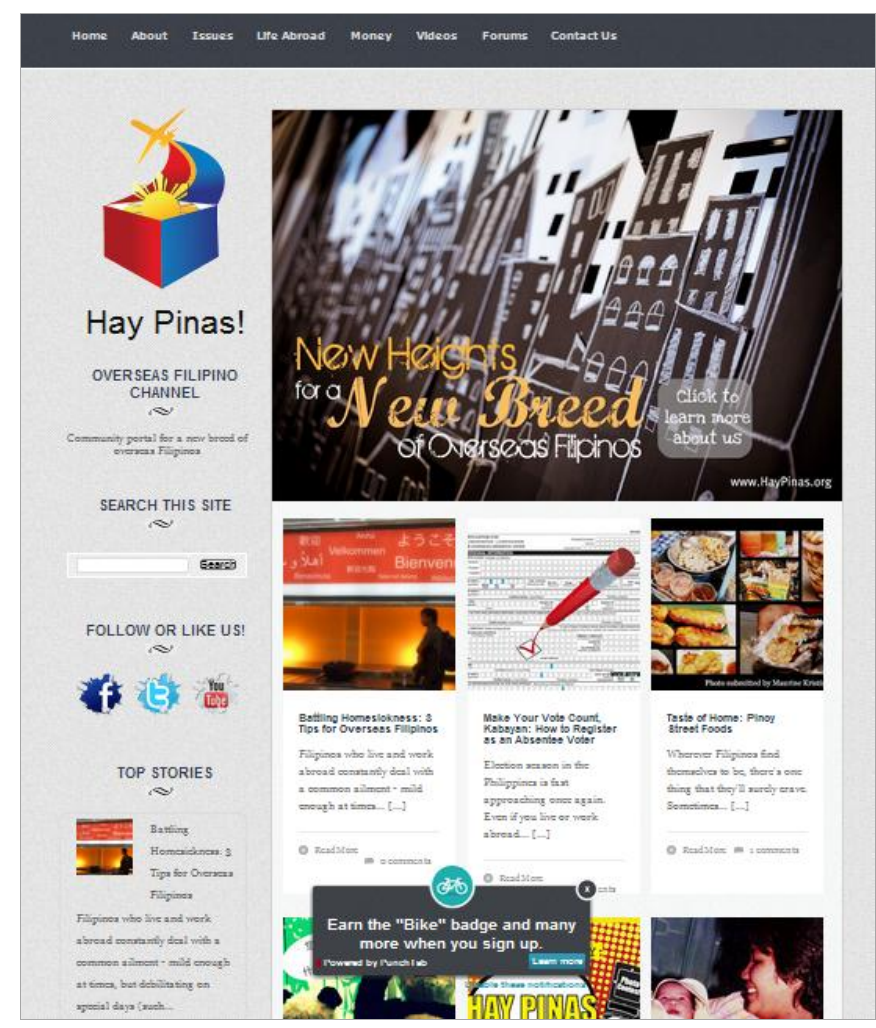

Figure 1: Screen capture of www.HayPinas.org dated September 5, 2012

To find out common migrant issues addressed by HayPinas.org to its target audience of overseas Filipinos, standalone articles on the website were categorized. Using the techniques of qualitative content analysis, the text data were systematically classified to identify and validate themes (Hsieh \& Shannon, 2005: 1278). Treating each of the 40 content pieces posted on the website from a one-year period as a unit of analysis, the articles were coded following these predetermined categories based on the expected information needs of migrant workers: "preparation and arrival", "adaptation", and "plan to return home”. These categories described the crossroads at every migrant's journey - from leaving the home country, adjusting and fitting in on the host society, and deciding whether to permanently stay or come back home 
someday. Articles that didn't fall on these categories were tagged as "other/miscellaneous" and were excluded from further evaluation. Also excluded from the analysis were the comments posted by users on the standalone articles. Table 1 presents the categories and its share of the total published content, and also the representative content samples based on the most popular content from web analytics.

Table 1: Content categories and representative popular posts

\begin{tabular}{|c|c|c|}
\hline Category & Percentage & Popular content based on web analytics \\
\hline Preparation and arrival & $35 \%$ & $\begin{array}{l}\text { - Video tutorial of required documents to prepare } \\
\text { before leaving the country for overseas work } \\
\text { - Smart phone apps for overseas Filipinos } \\
\text { - Advice to new graduates who might plan to go } \\
\text { abroad }\end{array}$ \\
\hline Adaptation & $51 \%$ & $\begin{array}{l}\text { - Video reaction to portrayal of overseas Filipinos } \\
\text { in local advertisement } \\
\text { - How to cope when there is a language barrier } \\
\text { - Practicing faith/religion while abroad }\end{array}$ \\
\hline Plan to return home & $14 \%$ & $\begin{array}{l}\text { - Importance of financial planning in the context } \\
\text { of migrant work } \\
\text { - Conquering the fear of managing finances } \\
\text { - Budgeting tips when planning short visits and } \\
\text { vacations to the Philippines }\end{array}$ \\
\hline
\end{tabular}

On its pilot year, content on the HayPinas.org website received 12,996 views (an average of 1,083 monthly page views with around $10 \%$ from returning visitors), while the 5 videos posted 
on the YouTube channel collectively logged 5,650 views. Among referring websites to HayPinas.org, organic searches from search engines represent the largest share. Web analytics data recorded the following top five key search terms that led users to the website: "Filipino channel", "balik-bayan (migrant returnee) inspirational quotes", "direct hiring for OFW", “financial literacy for OFW" and "OFW help". Except for the search term "Filipino channel", the keywords represented each stage of overseas Filipinos' perceived information needs - with content pointing to pre-employment requirements, investment options for remittances, technology lifelines to use in case of distress, and quotes to inspire homecoming. With the majority of audiences landing on HayPinas.org content from search results, it also suggested that the just-in-time nature of online media was an ideal platform for migrants. This mode of access also supported Del Rosario's observation that overseas Filipinos sought migration-related information on an as-needed basis (2007: 235). Other key traffic drawers to HayPinas.org content were from Facebook shares, link referrals from third-party blogs, and direct visits.

Although website content was mostly accessed through computers (88\%), use of mobile devices such as tablets and smart phones was also notable (12\%). By also offering a mobile version of its website, HayPinas.org ensured that audiences who access content on smaller device screens would have a convenient experience. But the choice was not without a tradeoff since navigation paths to HayPinas.org's social media channels, forums, and news feeds were not supported. In terms of demographics, audiences from countries with high concentrations of migrant Filipinos figured in the top audience sources: United States, Saudi Arabia, United Arab Emirates, Canada, and the United Kingdom. Excluding Taiwan and the Philippines where majority of the community members came from, rounding up the top ten countries were South Korea, Ukraine and Germany. A subscriber to the Google AdSense program, ad revenue 
paid for the renewal of HayPinas.org's domain registration and management for another year. By discussing the issues and interests of overseas Filipinos through online content, HayPinas.org championed the social group into protecting their rights, finances, and relationships as they leave for and live in other countries.

\section{Lessons Learned: Developing Responsive Content for Migrants}

As deduced from the content categories in HayPinas.org, the general information needs of migrants span these stages: pre-departure, settling in, and return home. Taking into consideration these periods of information needs could result to content that migrants can use to make sense of their journey from their country of origin to new destinations. With an eye on these topics, online media tools and channels can then be evaluated to reach out to the target community. Since more developing countries institutionalize labor export policies to lure remittances (Brinkerhoff, 2006), it is beneficial that migrants know how to access and produce online content to cope in foreign lands and cultures. With added data from the personal account of the HayPinas.org founder, the following are takeaways from the pilot year experience of the community portal.

a. Identify objectives and integrate online accounts. Aside from the website domain and content publishing platform, social media accounts were simultaneously created during the planning stages of HayPinas.org. Accounts were opened in YouTube, Facebook, and Twitter because of the popularity of these social media channels among overseas Filipinos. These content channels were assigned specific objectives. The website - regularly updated with blog articles and multimedia content - was considered as the central content gateway for driving visitors from search engines. YouTube hosted the video content that was embedded on the website. Social network accounts on Facebook and Twitter were relied 
on to promote content (both created by HayPinas.org and links to other resources) and to encourage interaction among community members. Since a common administrator profile was used to set up these accounts, the content channels linked to each other before HayPinas.org was launched.

b. Plan a content calendar. HayPinas.org maintained an editorial calendar that covered a period of one year to organize its content development goals. The content calendar plotted the publishing schedules, themes, content topics, and author responsibilities. Apart from conceptualizing the standalone content pieces, some social media prompts were also prepared to draw reaction from the online community. While the content calendar was useful especially in developing topics tied to special occasions and holidays, flexibility in prioritizing content was deemed as equally important. It was thus necessary to regularly review and update the editorial calendar. News and current affairs that related to the target audience were monitored and noted on the calendar as well. Based on HayPinas.org's web analytics data, companion articles and video responses to popular news items in mainstream media garnered some of the highest content views.

c. Define ideal and broad target audiences. Warschauer and Grimes (2007) identified two general types of Web 2.0 audiences: the immediate (those directly addressed by the content) and the virtually unlimited (anyone with Internet access). HayPinas.org's mission-statement explicitly described its ideal audience as Filipino professionals who have temporarily settled or permanently resided in other countries. Regular visits and comments received from outside of this target demographic (for example, from prospective migrants still based in the Philippines) deterred HayPinas.org's criteria of success at the onset. HayPinas.org then actively refocused its content within the perimeters of its target 
demographic (such as toning down a slant on human rights to concerns on cultural adaptation). But this seemed to be a countertenor to its wider, unlimited audience. Although maintaining a consistent tone and voice proved instructive, it became obvious to HayPinas.org that it had to continue talking about the larger context of labor migration.

d. Drive cross-platform content consumption. To maximize the exposure of HayPinas.org's content, posts were interlinked with related content where possible and re-shared on its social network accounts. YouTube video uploads were similarly republished as blog posts. Though the social network accounts in Facebook and Twitter were initially conceived to cross-promote content from the website and YouTube account, HayPinas.org recognized the need to develop specific content for these channels to drive more engagement from its online community. This was in line with Kaplan and Haenlein's (2010) advice that it was best to use different contact points so long as social media activities were aligned with each other. HayPinas.org separately posted polls and inspirational quotes on social networks, which received a favorable response. It also hosted an amateur photo contest, calling out its community members to post photos that remind them of home on its Facebook page, the winning entry of which was turned into a blog post.

e. Collect and respond to user data, such as search queries. Aside from readily evident user data such as comments and email responses to published content, HayPinas.org assessed built-in statistics from its web host and from a third-party website tracking counter. Search queries that led visitors to HayPinas.org were evaluated. Reviewing keywords ensured that correct labels drive users to landing on HayPinas.org content and that user-generated keywords call out possible future content to work on. For example, since there was considerable number of visits from the keyword "financial literacy for OFW", a series 
about financial planning had been consequently developed, along with a dedicated website tab. The navigation paths that users took and their corresponding actions upon landing on a particular page - such as if they stayed on specific content longer, left or exited a page after a few seconds, or clicked on links to related content - were also telling of users' information-seeking behaviors and level of engagement.

Building on these lessons learned, HayPinas.org followed a four-phased content development cycle: "conceptualize", “create", "cross-promote" and "canvass". The model below illustrates the process:

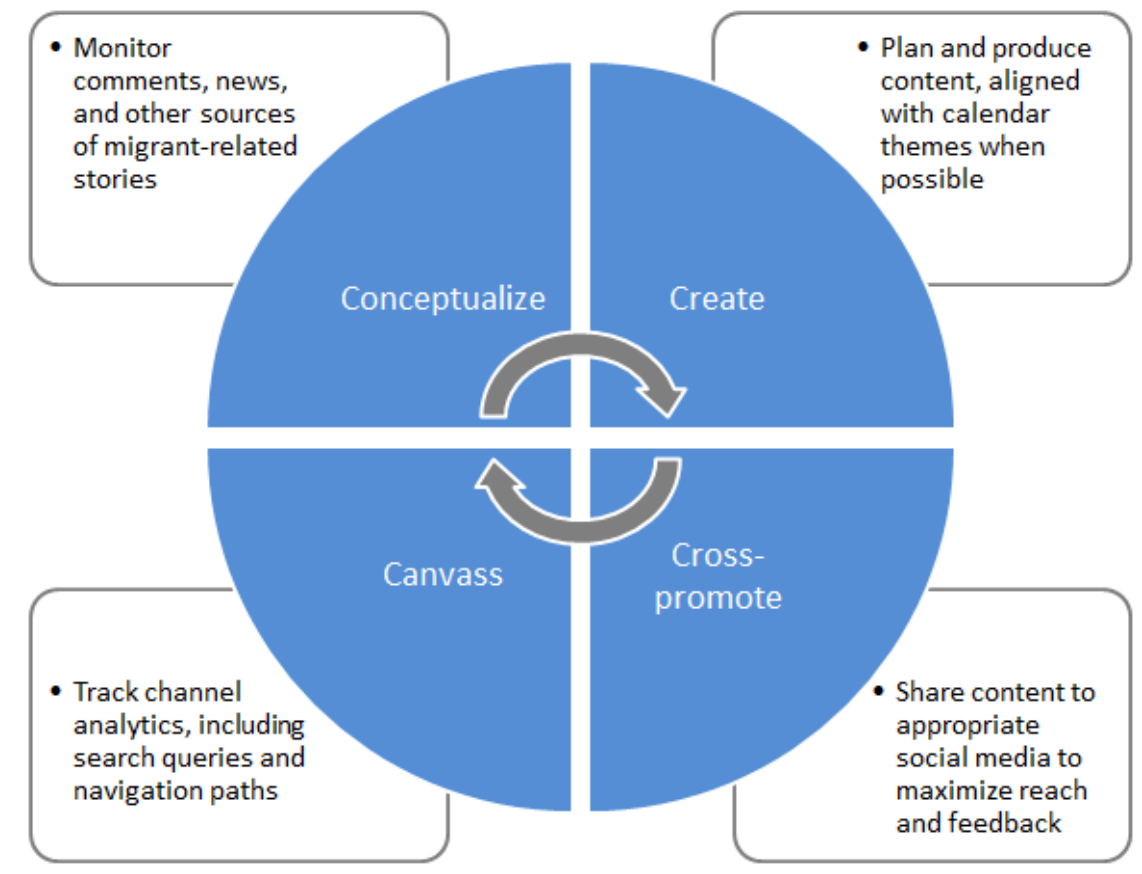

Figure 2: HayPinas.org content development model

The first phase in HayPinas.org's content development cycle is to conceptualize content ideas. Previous comments, related news developments, and questions by the migrant Filipinos commonly found in social networks are good sources of content ideas. Regular monitoring of these sources is needed to keep up with contemporary issues. Since the pool of contributors 
are overseas Filipinos themselves, personal experience and observations served as a wellspring of content topics. During the content creation phase, it is recommended to align the angle of the content with pre-determined editorial themes and calendar schedules. Philippine national holidays and commemorations (for example, National Heroes Day) and popular international events (for example, Christmas) serve as helpful pegs for scheduling the content posting of themed articles. Flexibility must also be factored in to accommodate other types of content. The goal is to both be timely and timeless, since the content does not follow a traditional journalistic cycle.

When a content asset (text/photo/video) is published on the main content platform (the website, in this case), the next stage is to promote it on relevant channels. Within the originating channel itself, tags and labels must be clearly defined and search-engine optimized for better findability. When cross-posting content to other channels (for example, social networks), it is best to consider unique channel requirements (such as word count limitation) and audience expectations. Related copy may be tailor-fitted to suit other channels, especially social networks. The last phase of the content development cycle is to canvass or evaluate the content performance in the channels where it content is posted on. Some metrics to track include views (outreach), visit duration, comments, and interactions (engagement), and re-shares on social networks (amplification) but measuring these figures need to be tied to certain goals. Data from analytics is also a good idea pool of new content to develop.

\section{Concluding Discussion}

From reviewing available data on its pilot year, HayPinas.org is on track in producing and delivering niche content to its target audience of overseas Filipinos. Its content has covered relevant themes concerning the Filipino diaspora, namely preparing to leave the country, 
settling in and adapting as a migrant on the host country, and planning to return home. Future web redesign could consider grouping content into these themes. Though text-based articles composed the majority of content that HayPinas.org has created, videos proved to yield more visitor views and engagement. This, along with web analytics data that showed an increasing number of the target audience using mobile devices to access digital content, further pointed to the trend of a converging media environment. HayPinas.org's use of popular social networks has also enabled it to disseminate content to a wider audience and to also interact with them. Though limited to a single-site case study and is therefore not generalizable, HayPinas.org's process model has proven responsive to its target audience of migrant Filipinos because it emphasized monitoring their information demands (such as from search queries), ensured that content can be found on search engines (through tags and keywords) and shared on social networks, and funneled back the user data onto its content development cycle.

The community that HayPinas.org's social media content harbors may be considered as cohesive because of their shared interests and information needs. However, a "critical mass" of users who interact regularly is needed to sustain any web-based community (Jones, Ravid, \& Rafaeli, 2004). To better succeed at its mission, HayPinas.org should be able to convert more website visitors into community members. Aside from word-of-mouth referrals, some strategies it could employ include email marketing, online advertisements, and partnerships with popular content providers. More content should be rolled out to increase visits from organic searches. As it builds on successes from its pilot year, HayPinas.org could start focusing on user-generated content (comments, forum posts, and contributions) as an important resource for its target audience. It should also be useful to its community if region-specific concerns, especially from countries where it has sizable audiences, would be addressed. Follow-up studies on audience reception and process model evaluation by content 
producers with a similar agenda are suggested. Migrants - many of whom are treated as outsiders and without means to echo their questions, interests, and personal narratives in mainstream media - can reclaim a voice and rekindle a sense of belongingness through social media. 


\section{References}

Ayalon, L. (2009). "Evaluating the working conditions and exposure to abuse of Filipino home care workers in Israel: characteristics and clinical correlates.” International Psychogeriatrics, 21(1): 40-49.

Bowman, B. S., \& Willis, C. (2003). We Media: How Audiences are Shaping the Future of News and Information. The Media Center at the American Press Institute, Reston, VA. Retrieved 23 August 2006 from http://mediacenter.org/mediacenter/research/wemedia

Brinkerhoff, J. ( 2006). “Diasporas, Skills Transfer, and Remittances: Evolving Perceptions and Potential" in Converting Migration Drains into Gains, C. Wescott and J. Brinkerhoff (eds.). Manila: Asian Development Bank.

Castells, M., \& Cardoso, G. (2005). The Network Society: From Knowledge to Policy. Washington DC: Center for Transatlantic Relations.

Commission on Filipinos Overseas. (2010). Stock Estimate of Overseas Filipinos. Retrieved on 15 March 2012 from http://www.cfo.gov.ph/pdf/statistics/Stock\%202010.pdf.

Del Rosario, J. (2007). "Information Needs of Filipinos in Belgium: A Survey Research" in In De Old World: Views of Filipino Migrants in Europe, F. Hoegsholm (ed.). Quezon City: Philippine Migration Research Network and Philippine Social Science Council.

Figer, R. (2009). “I am P/Ninoy: Filipino Diaspora, Technology and Network Nationalism.” Journal of Global Communication, 2(1), 264-278.

Figer, R., \& De Torres, C. (2012). “Technology and Diaspora: Investigating Immigration Issues in Timog Online." Online Journal of Communication and Media Technologies, 2(2), 79-102.

Go, S. (2003). "Recent Trends in Migration Movements and Policies: The Movement of Filipino professionals and Managers" in Migration and the Labour Market in Asia. Paris: OECD Publications. 
Herring, S. C. (2004). "Slouching Toward the Ordinary: Current Trends in Computer-Mediated Communication.” New Media \& Society, 6(1), 26-36.

Hartley, J. (2002). Communication, Cultural and Media Studies: The Key Concepts. London: Routledge.

Hsieh, H., \& Shannon, S. (2005). “Three Approaches to Qualitative Content Analysis.” Qualitative health research, 15(9), 1277-88.

Jones, Q., Ravid, G., \& Rafaeli, S. (2004). “Information Overload and the Message Dynamics of Online Interaction Spaces: A Theoretical Model and Empirical Exploration.” Information Systems Research, 15(2), 194-210.

Kaplan, A. \& Haenlein, M. (2010). "Users of the World, unite! The Challenges and Opportunities of Social Media.” Business Horizons, 53(1), 59-68.

Klinenberg, E. (2005). “Convergence: News Production in a Digital Age.” The Annals of the American Academy of Political and Social Science, 597(1), 48-64.

Komito, L., \& Bates, J. (2011). “Migrants' Information Practices and Use of Social Media in Ireland: Networks and Community.” Proceedings of the 2011 iConference, 289-295. New York: Association for Computing Machinery.

Mehra, B., Merkel, C., \& Bishop, A. (2004). “The Internet for Empowerment of Minority and Marginalized Users". New Media \& Society, 6(6), 781-802.

Ofreneo, R. \& Samonte, I. (2005). Empowering Filipino Migrants: Policy Issues and Challenges. Geneva: International Labor Organization.

Orange, G., Seitz, V. \& Kor, A. (2012). “Information Dissemination Needs of Indonesian Migrant Domestic Workers in Malaysia.” Journal of Southeast Asian Research, 2012: $1-16$.

Paragas, F. (2006). Eccentric Networks: Patterns of Interpersonal Communication, Organizational Participation, and Mass Media Use among Overseas Filipino Workers. 
Doctoral dissertation, Ohio University. Retrieved on 30 July 2012 from http://rave.ohiolink.edu/etdc/view?acc_num=ohiou1147119861

Preston, P., \& Kerr, A. (2001). "Digital Media, Nation-states and Local Cultures: the Case of Multimedia 'Content' Production.” Media, Culture \& Society, 23(1), 109-131.

Warschauer, M. and Grimes, D. (2007). “Audience, Authorship, and Artifact: the Emergent Semiotics of Web 2.0.” Annual Review of Applied Linguistics 27, 1-23.

Yao, A. (2009). "Enriching Migrant Experience: Blogging Motivations, Privacy and Offline Lives of Filipino Women in Britain”, First Monday, Vol. 14: 3. Retrieved on 15 March 2012 from http://firstmonday.org/htbin/cgiwrap/bin/ojs/index.php/fm/article/view/2163\%40article $/ 2100$ 